\title{
Mast Cell Infiltration and Leukotriene Receptor Expression in Various Tumors: Possible Clinical Application of Common Pathological Findings Concerned with Tumor Development
}

\author{
Masao Sugamata*, Tomomi Ihara, Miho Sugamata \\ Department of Pathology, Tochigi Institute of Clinical Pathology, Tochigi, Japan \\ Email: "mspathol@beige.ocn.ne.jp
}

Received 18 June 2015; accepted 18 July 2015; published 21 July 2015

Copyright $\odot 2015$ by authors and Scientific Research Publishing Inc.

This work is licensed under the Creative Commons Attribution International License (CC BY). http://creativecommons.org/licenses/by/4.0/

c) (i) Open Access

\section{Abstract}

Since the mechanisms of the developmental processes of tumors remain unclear, early detection and early treatment of the tumors is necessary to save patients with malignant tumors. Therapies currently available to patients are surgery, chemotherapy, and radiotherapy. However, there are many patients who cannot be saved by such therapies. In this study, we found the common features of various tumor tissues, and we demonstrated the effect of therapeutics that target them by using experimental rats with spontaneous tumors. 26 kinds of human tumors (epithelial or mesenchymal origin, and malignant or benign) and Sprague-Dawley rats with spontaneous mammary gland tumors were examined by light and electron microscope. To detect of mast cells and leukotriene receptor, toluidine blue stain and immunohistochemical stain were performed. The rats were orally administered one of leukotriene receptor antagonists. We found that the presence of numerous mast cells and expression of leukotriene receptors in various tumor (human tumors and rat spontaneous tumors). And the therapeutic effects, which suppressed not only tumor progress but also angiogenesis and nerve formation, for rat tumors by administration of leukotriene receptor antagonist could obtain. Our data suggest the possibility that allergic reactions, in which leukotriene and leukotriene receptors in particular appear to play an important role, are involved in the development and progression of tumors and that it may be possible to control tumor progression by regulating such reactions. Our results might be useful for clinical applications of oncotherapy.

${ }^{*}$ Corresponding author.

How to cite this paper: Sugamata, M., Ihara, T. and Sugamata, M. (2015) Mast Cell Infiltration and Leukotriene Receptor Expression in Various Tumors: Possible Clinical Application of Common Pathological Findings Concerned with Tumor Development. Journal of Cancer Therapy, 6, 606-612. http://dx.doi.org/10.4236/jct.2015.67066 


\section{Keywords}

\section{Mast Cell, Leukotriene Receptor, Anti-Leikotriene Therapy, Apoptosis}

\section{Introduction}

At present, the therapeutic options for malignant tumors include surgery, chemotherapy, and radiotherapy. However, many patients cannot be saved by these therapies. In many cases of a suspected benign tumor, the approach is to simply observe the progress of the tumor, and the tumor is then removed surgically when considered necessary. The patient then undergoes regular medical examinations for early discovery of malignant alterations to the tumor. Since the mechanisms and developmental processes of tumors remain unclear, this approach seems to represent the most effective means for achieving early detection of malignancy. However, a more effective method of saving patients with malignant tumors would be the early detection and early treatment of such tumors.

We recently discovered that mast cells are strongly involved in the development of endometriosis [1] [2]. An allergic-type reaction appears to lead to lesion development, which presents as a proliferation of ectopic endometrial-like cells. We have previously shown that anti-leukotriene therapy, which is an anti-allergy (especially, asthma) therapy, is very effective in treating endometriosis [3] [4]. Those studies showed that the presence of leukotrienes and their receptors is a factor in the development of an endometriosis lesion. Although endometriosis is a benign disease, there is a large increase in ectopic endometrial cell proliferation and the amount of interstitial tissue increases; these characteristics resemble those of a malignant tumor. We therefore considered that, from this perspective, the processes that underlie the progression of malignant tumors and endometriosis might be very similar.

In this study, we confirmed the expression of leukotriene receptors, which were expressed as part of an allergic reaction, in various human tumor tissues and we discovered that this pathological finding was common to a range of tumors. We further demonstrated the therapeutic effect of targeting leukotriene receptors by treating spontaneous tumors of experimental animals.

\section{Materials and Methods}

\subsection{Rat Spontaneous Tumors and Light Microscopic Analysis}

Sprague-Dawley (SD) rats (all rats are females, mean weight: $605.62 \pm 38.38 \mathrm{~g}$ ) that had spontaneous mammary gland tumors were purchased from Charles River Laboratories Japan, Inc. (Kanagawa, Japan).

\subsubsection{Histopathology and Localization of Mast Cells}

Tissue samples from the tumor area and a non-tumor area (for example, a left mammary gland was obtained as the comparison (non-tumor area) if the mammary gland which a tumor existed in was a right mammary gland) of the anesthetized SD rats with and without anti-leukotriene therapy, were fixed with $10 \%$ buffered formalin, and, after routine dehydration, were embedded in paraffin. Sections $5-\mu \mathrm{m}$ thick were stained with hematoxylin and eosin (HE) and examined under a light microscope.

To identify mast cells in each specimen, the paraffin sections were stained with toluidine blue. The granules within mast cells contain heparin and sulfated glycosaminoglycan that stain metachromatically with toluidine blue. The $5-\mu \mathrm{m}$ tissue sections were stained for 30 minutes with a staining solution containing a $0.05 \%$ concentration of toluidine blue O (Kanto Chemical Co., Inc., Tokyo, Japan) in a citric acid phosphate buffer (pH 2.5), and were then examined by light microscopy.

\subsubsection{Detection of Leukotriene Receptors Using Immunohistochemical Staining}

Immunohistochemical staining for the cysteinyl leukotriene receptor (CysLT) 1 and 2 was performed to detect the expression of the leukotriene receptors in the tumors under a light microscope. The $5-\mu \mathrm{m}$ tissue sections were stained immunohistochemically using the streptavidin-biotin method (Histofine SAB-PO Kit; Nichirei, Tokyo, Japan). The primary antibodies used were polyclonal antibody to CysLT1 and polyclonal antibody to 
CysLT2 (Acris Antibodies, Inc., San Diego, CA, USA).

\subsection{Animals and Anti-Leukotriene Therapy for Rat Spontaneous Tumors}

The rats were orally administered one of the following leukotriene receptor antagonists: Zafirlukast (Astra Zeneca, London, UK), Monterukast sodium (Merck, NJ, USA), Pranlukast hydrate (Ono Pharmaceutical Co., Ltd., Osaka, Japan) or Zileuton (Abbott Laboratories; Chicago, IL, USA). The experiment dose of each medicine was decided referring to the amount of prescription (to the adult asthmatic) that it was mentioned in each attached document. The medication periods for each medicine were for 3 or 7 days (Zafirlukast $(1.33 \mathrm{mg} / \mathrm{kg}$ ); the one oral administration/day; total three days $(\mathrm{n}=3)$ or seven days $(\mathrm{n}=3)$, Monterukast sodium $(0.16 \mathrm{mg} / \mathrm{kg}$ per day); the one oral administration/day; total three days $(\mathrm{n}=3)$ or seven days $(\mathrm{n}=3)$, Pranlukast hydrate $(7.5 \mathrm{mg} / \mathrm{kg}$ per day); the one oral administration/day; total three days $(n=3)$ or seven days $(n=3)$, Zileuton $(34.3 \mathrm{mg} / \mathrm{kg}$ per day); the one oral administration/day; total three days $(n=3)$ or seven days $(n=3)$ ). Four non-treated female rats (distilled water $(0.5 \mathrm{ml})$, the one oral administration/day, total three days $(\mathrm{n}=2)$ or seven days $(\mathrm{n}=2)$ ) that had spontaneous tumors were used as comparative controls.

All experimental animals were handled in accordance with institutional and national guidelines for the care and use of laboratory animals.

\subsection{Human Tumors and Light Microscopic Analysis}

Tumor tissues obtained from patients who were diagnosed with various tumors, which were confirmed based on the standard for diagnosis of the Japanese society of surgical pathology, are listed in Table 1 . Tumors were sampled after the patients gave their informed consent in accordance with the Helsinki declaration. To identify mast cells and detect the presence of leukotriene receptors in the tumor tissue, toluidine blue $\mathrm{O}$ staining and immunohistochemical staining for CysLT 1 and 2 were performed in a similar manner to the staining of the tumor tissues of the rats.

\subsection{Electron Microscopic Analysis}

The tissue samples were immediately fixed with $1 \%$ glutaraldehyde and $4 \%$ formalin for 6 hours at $4^{\circ} \mathrm{C}$ and were then rinsed in $0.1 \mathrm{M}$ cacodylate buffer overnight. The tissues were postfixed with $1 \%$ osmium tetroxide and were embedded in Epon 812 resin. Ultrathin sections were prepared using an Ultramicrotome (Model MT-XL; RMC, Arizona, USA), were doublestained with uranyl acetate and lead citrate, and were then examined under an electron microscope (Model JEM1400; JEOL, Tokyo, Japan).

Apoptotic appearance was evaluated based on the characteristics of an ultrastructural process in the apoptotic body, as described in our previous report [5]. In brief, the ultrastructural process of apoptosis is divided into four stages, and characteristic findings can be observed at each stage. One such finding is the space described as a crescent-shaped space (CSS). A CSS appears around the nuclear membrane of a cell from the first stage of the morphologic process of apoptotic body formation. Although a cell may appear normal in other respects, when a CSS appears, apoptosis may be induced.

\section{Results}

We first checked for the presence of mast cells in the tissue specimens prepared from 26 kinds of human tumors (epithelial or mesenchymal origin, and malignant or benign tumors such as gastric carcinoma, colon cancer, esophagus epidermoid carcinoma, breast cancer, prostatic cancer, liver cancer, gastrointestinal stromal tumor, leiomyosarcoma, rhabdomyosarcoma and myoma uteri). In all tumor samples analyzed numerous mast cells were detected. These mast cells were diffusely distributed within the tumor, and such a diffuse distribution was very similar to that of mast cells in the lesion of the endometriosis patient that we previously studied [Figure 1(a), Figure 1(b)].

We then used Sprague-Dawley rats with spontaneous mammary gland tumors (which, as expected, displayed many mast cells within the tumors [Figure 1(c)]) to investigate the anticancer efficacy of anti-leukotriene therapy, which is a pharmacotherapy for bronchial asthma that has proven very effective against endometriosis [3] [4]. In addition, immunohistochemical staining for CysLTs was performed to determine which of these receptors 
Table 1. List of human tumors.

\begin{tabular}{|c|c|c|}
\hline Malignant tumors & \multicolumn{2}{|c|}{ Number of specimen } \\
\hline \multicolumn{3}{|l|}{ Epithelial origin } \\
\hline \multirow[t]{2}{*}{ Pharyngeal carcinoma (Squamous cell carcinoma) } & Well & $\mathrm{N}=2$ \\
\hline & Poorly & $\mathrm{N}=2$ \\
\hline Esophageal carcinoma (Squamous cell carcinoma) & Moderately & $\mathrm{N}=2$ \\
\hline Gastric cancer & Poorly & $\mathrm{N}=5$ \\
\hline \multirow[t]{3}{*}{ Colon cancer (Adenocarcinoma) } & Well & $\mathrm{N}=5$ \\
\hline & Moderately & $\mathrm{N}=3$ \\
\hline & Poorly & $\mathrm{N}=3$ \\
\hline Liver cancer (Hepatoblastoma) & & $\mathrm{N}=3$ \\
\hline (Hepatocellular carcinoma) & & $\mathrm{N}=4$ \\
\hline \multirow[t]{2}{*}{ Gallduct carcinoma (Adenocarcinoma) } & Well & $\mathrm{N}=3$ \\
\hline & Poorly & $\mathrm{N}=3$ \\
\hline Renal cell carcinoma & & $\mathrm{N}=5$ \\
\hline \multirow[t]{3}{*}{ Pancreatic carcinoma (Adenocarcinoma) } & Well & $\mathrm{N}=5$ \\
\hline & Moderately & $\mathrm{N}=3$ \\
\hline & Poorly & $\mathrm{N}=3$ \\
\hline Breast cancer (Intraductal carcinoma; noninvasive) & & $\mathrm{N}=5$ \\
\hline (Scirrhus carcinoma; invasive) & & $\mathrm{N}=3$ \\
\hline Prosstatic carcinoma (Adenocarcinoma) & & $\mathrm{N}=5$ \\
\hline Thyroid gland carcinoma (Papillary carcinoma) & & $\mathrm{N}=3$ \\
\hline \multicolumn{3}{|l|}{ Mesenchymal orgin } \\
\hline Rhabdomyosarcoma & & $\mathrm{N}=2$ \\
\hline Leiomyosarcoma & & $\mathrm{N}=2$ \\
\hline Hemangiosarcoma & & $\mathrm{N}=2$ \\
\hline Total number of malignant tumors: 22 & \multicolumn{2}{|c|}{ Total of specimen: 73} \\
\hline Benign tumors & \multicolumn{2}{|c|}{ Number of specimen } \\
\hline \multicolumn{3}{|l|}{ Epithelial origin } \\
\hline Schwannoma & & $\mathrm{N}=2$ \\
\hline Pituitary adenoma & & $\mathrm{N}=4$ \\
\hline Follicle adenoma (Thyroid gland) & & $\mathrm{N}=3$ \\
\hline \multicolumn{3}{|l|}{ Mesenchymal orgin } \\
\hline Myoma uteri & & $\mathrm{N}=15$ \\
\hline Total number of benign tumors: 4 & \multicolumn{2}{|c|}{ Total of specimen: 24} \\
\hline
\end{tabular}



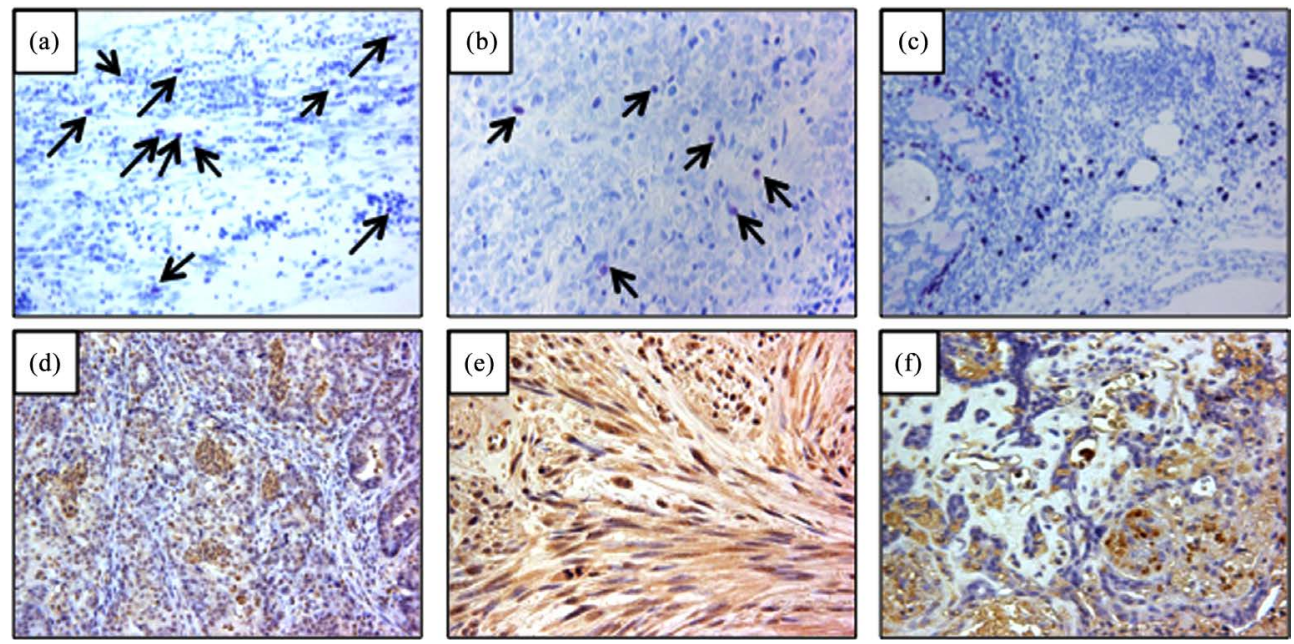

Figure 1. Pathological findings of human and rat tumors. (a)-(c) Detection of mast cells in human and rat tumors by toluidine blue staining. Positively stained cells (Violet color) are mast cells; (a) Representative human gastric carcinoma (Mag: 400×) (arrow: mast cell); (b) Representative human leiomyosarcoma (Mag: 400×) (arrow: mast cell); (c) Representative Sprague Dawley (SD) rat spontaneous mammary gland tumor (Mag: 200×); (d)-(f) Detection of cysteinyl leukotriene receptor (CysLT) expression in human and rat tumors by immunohistochemical staining. The brown color denotes positive staining; (d) Representative human gastric carcinoma (Mag: 400×); (e) Representative human leiomyosarcoma (Mag: 400×); (f) Representative SD rat spontaneous mammary gland tumor (Mag: 400×).

was expressed in human and rat tumor tissues, and thus, which of these receptors react with the LT-R antagonists that were administered to the rats. CysLT-positive cells that were diffusely distributed within the tumor tissue were observed in all of the rat spontaneous tumors. Positive reactivity to the anti-CysLT antibodies was detected not only in tumor cells, but also in fibroblasts, mast cells, and endothelial cells [Figure 1(f)]. All of the human tumor tissues that were checked at this time (both malignant and benign) also showed expression of these receptors similar to the rat tumors [Figure 1(d), Figure 1(e)].

Under light microscopy, apoptotic cells were detected in leukotriene receptor antagonist-treated rat tumors. These apoptotic cells were diffusely distributed throughout the tumor, and were not detected in the non-treated group [Figure 2(a)]. Under electron microscopy, apoptotic cell death including crescent-shaped spaces (CSSs) was induced not only in tumor cells, but also in interstitial cells (fibroblasts, endothelial cells and smooth muscle cells of vessels) [Figures 2(b)-(e)].

Furthermore, alterations in the nervous tissue of tumor tissues were observed that varied according to each leukotriene receptor antagonist administered. In rat tumors that were treated with Zafirlukast or Monterukast sodium, destruction of the myelin sheath was observed, although no degeneration of the axon was seen [Figure 2(f), Figure 2(g)]. The Pranlukast hydrate-treated group showed severe degeneration of axons although only slight myelin destruction was seen [Figure 2(h)], and, in the Zileuton-treated group, only degenerative change of axons was observed [Figure 2(i)]. These findings were common to all of the rat tumors from each respective leukotriene receptor antagonist-treated group. No marked changes were seen in paired adjacent tissues (nontumor areas) or non-treated tumor tissue.

\section{Discussion}

In various human tumors and in the rat spontaneous tumors, the presence of numerous mast cells and the expression of leukotriene receptors were common features of tumors of epithelial or mesenchymal origin, whether they were malignant or benign tumors. These pathological findings show that anti-leukotriene therapy has a therapeutic effect against spontaneous tumors in rats. This is the first report of such findings.

Moreover, the results suggest a lack of side effects of this treatment, since no apoptotic effects or adverse effects on nervous tissue were evident in non-tumor areas. The effects of the leukotriene receptor antagonists were 

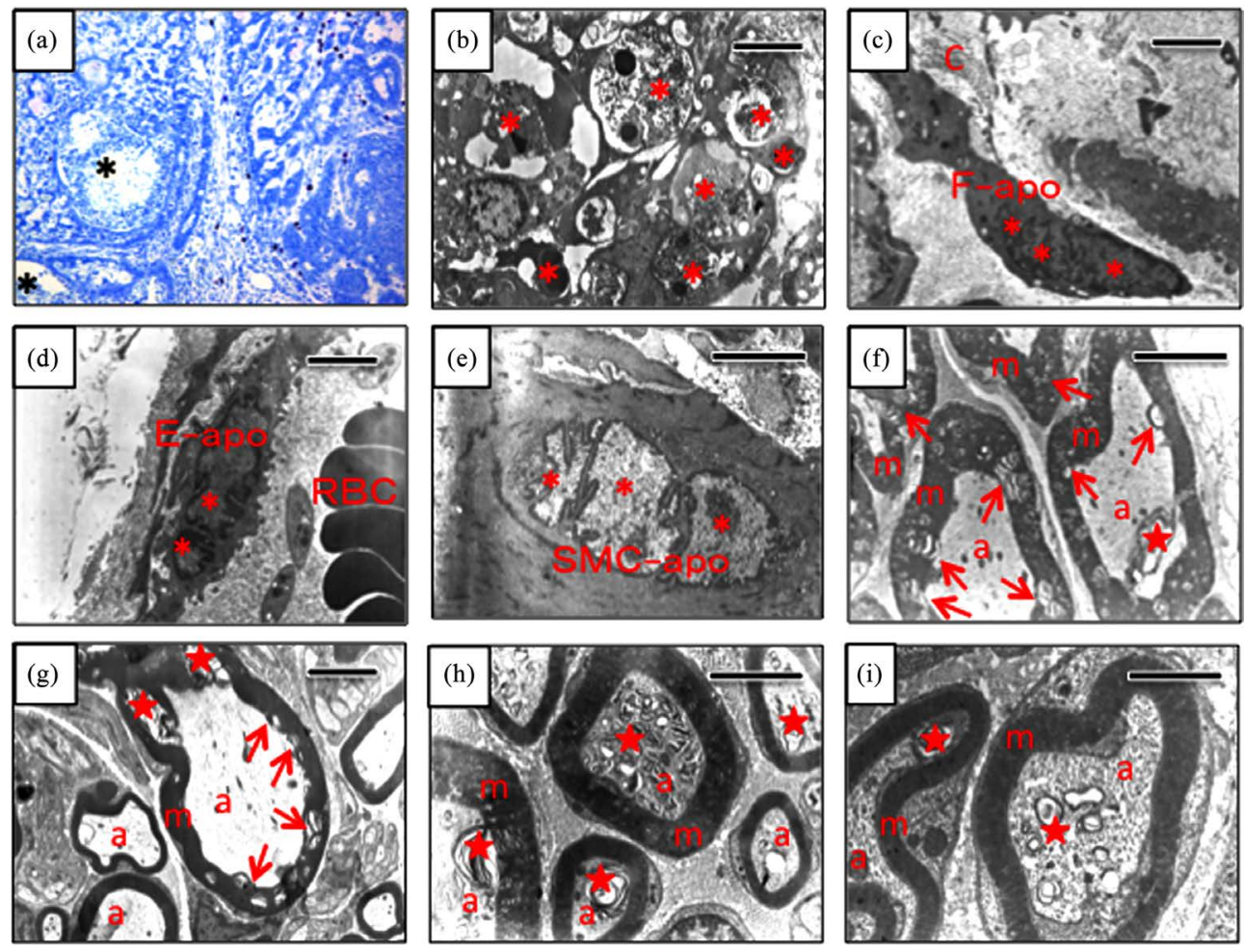

Figure 2. Therapeutic effect of leukotriene receptor antagonists against spontaneous rat tumors, analyzed using light and electron microscopy. (a) Light microscopic observation of a representative rat tumor (Toluidine blue stain) (Mag: 200×). Many apoptotic cells, which were presumed to be tumor cells, were detected within the tumor tissue, with a diffuse distribution (*); (b)-(e) Electron microscopic observations of apoptosis. (Double stain of uranyl acetate and lead citrate) Scale bar indicates $3 \mu \mathrm{m}$. Apoptotic cell death was induced not only in tumor cells [(b): *, apoptotic tumor cell] but also in interstitial cells that were fibroblasts [(c): F-apo, apoptotic fibroblast; *, nuclear fragmentation; C, a collagen fiber] and in endothelial cells [(d): E-apo, apoptotic endothelial cells, *, nuclear fragmentation; RBC, red blood cell.] and smooth muscle cells [(e): SMC-apo, apoptotic smooth muscle cell, *, nuclear fragmentation] of blood vessels; (f)-(i) Electron microscopic observation of nervous tissue within the tumor tissue. (Double stain of uranyl acetate and lead citrate) Scale bar indicates $3 \mu \mathrm{m}$. Destruction of the myelin sheath (arrow) and degeneration of the axon ( $\star$ ) were seen. (a, axon; m, myelin sheath.) (f) Zafirlukast-treated group. (g) Monterulast sodium-treated group. (h) Pranlukast hydrate-treated group. (i) Zileuton-treated group.

diverse. Thus, leukotriene receptor antagonist treatment not only affected tumor cells by inducing apoptosis, but inhibited angiogenesis by inducing apoptosis of endothelial cells and smooth muscle cells of blood vessels.

Furthermore, this treatment also inhibited neurogenesis in the tumor, presumably because the leukotriene receptors are diffusely distributed in the tumor tissue from rats with spontaneous tumors. Therefore, anti-leukotriene treatment may not only result in a decline in the amount of tumor tissue, but pain in terminal patients might also be eased. Although these data are very interesting, more data need to be accumulated in order to understand the mechanism of these effects since some differences were seen in the effect of each antagonist. In any case, since leukotriene receptors are also expressed in various human tumors, the present results obtained in rats suggest that similar effects in human patients might be expected.

The pathological findings regarding mast cells and leukotriene receptors that were obtained in this study of rat spontaneous tumors, also appeared to apply to human tumors, regardless of the kind of tumor. There is therefore a high possibility that allergic reactions including infiltration of mast cells are involved in the development and progression of tumors. In addition, as for the endometriosis lesion as well, we have already detected the existence of leukotriene receptor [6]. Therefore, it has the possibility that the leukotriene receptor exists in the various proliferative lesions including the tumor, and anti-leukotriene treatment may be effective in those diseases. 
In particular, leukotrienes and leukotriene receptors seem to play an important role in these allergic reactions and their regulation might therefore control tumor progression. Because our findings were observed in common with the benign tumor and the malignant tumor both, it may have the effect, which prevents a benign tumor from becoming malignancy or the recurrence of the malignant tumor. And, effect on metastasis inhibition will be anticipated because this therapy prevents angiogenesis and nerve formation, too. Anti-leukotriene treatment may not only have therapeutic effects on, but also preventive effects against tumors. Our data might be expected to lead to clinical applications of anti-leukotriene treatment in oncotherapy.

\section{References}

[1] Uchiide, I., Ihara, T. and Sugamata, M. (2002) Pathological Evaluation of the Rat Endometriosis Model. Fertility and Sterility, 78, 782-786. http://dx.doi.org/10.1016/S0015-0282(02)03327-7

[2] Sugamata, M., Ihara, T. and Uchiide, I. (2005) Increase of Activated Mast Cells in Human Endometriosis. American Journal of Reproductive Immunology, 53, 120-125. http://dx.doi.org/10.1111/j.1600-0897.2005.00254.X

[3] Ihara, T., Uchiide, I. and Sugamata, M. (2004) Light and Electron Microscopic Evaluation of Antileukotriene Therapy for Experimental Rat Endometriosis. Fertility and Sterility, 81, 819-823. http://dx.doi.org/10.1016/j.fertnstert.2003.08.029

[4] Ihara, T., Uchiide, I. and Sugamata, M. (2004) Light and Electron Microscopic Evaluation of Antileukotriene Therapy on Human Endometriosis. In: Daya, S., Pierson, R., Gundy, J., Eds., International Congress Series, Vol. 1271, Research Papers in Fertility and Reproductive Medicine, Elsevier BV, Amsterdam, 221-224. http://dx.doi.org/10.1016/j.ics.2004.05.165

[5] Ihara, T., Yamamoto, T., Sugamata, M., Okumura, H. and Ueno, Y. (1998) The Process of Ultrastructural Changes from Nuclei to Apoptotic Body. Virchows Archiv, 433, 443-447. http://dx.doi.org/10.1007/s004280050272

[6] Sugamata, M., Ihara, T. and Uchiide, I. (2015) A New Therapy for Human Endometriosis: The Therapeutic Value of Leukotriene Receptor Antagonist for Endometriosis. Open Journal of Obstetrics and Gynecology, 5, 313-318. http://dx.doi.org/10.4236/ojog.2015.56045 